\title{
A Cross Traffic Estimate Model for Optical Burst Switching Networks
}

\author{
Yujue WANG ${ }^{1}$, Dawei NIU ${ }^{2, ~ b}$, Weibo $\mathrm{YU}^{2,}$,, Hai WANG ${ }^{2, \mathrm{~d}}$, Zhichao MI $^{2, \mathrm{e}}$, \\ Dong CAO ${ }^{2, \mathrm{f}}$, Lei Zhang ${ }^{2, g}$ \\ ${ }^{1}$ Naval Academy of armmament, Beijing, 100001, China. \\ ${ }^{2}$ Institute of Communication Engineering of PLA University of Science and Technology, Nanjing, \\ 210007, China. \\ aemail: janejue@163.com, bemail: flyndw@ sina.com, cemail: \\ bigtouyu@163.net,_demail: haiwang@ieee.org, ${ }^{\mathrm{e} e m a i l: m i z h i c h a o @ 163 . c o m, ~}{ }^{\mathrm{f}} \mathrm{email}$ \\ Caodong@gmail.com, ${ }^{9}$ email:leizhang@163.com
}

Keywords: Estimate Model, Optical Burst Switching, Offset Time, Queuing Model.

\begin{abstract}
The cross traffic intensity of the optical burst switching network is a key parameter for the edge node operations. This paper provides an estimate model for edge nodes to infer the cross traffic on the control plane of the bottleneck core node. A normal M/D/1 and a special M/G/1 queuing model are applied to core nodes without and with wavelength conversion device respectively. Service time of the special M/G/1 queuing model is dependent with the traffic load. Edge nodes can then estimate the cross traffic of the core node through a likelihood function as well as a moment estimator. The observing variable that input to the estimator is the sojourn time of the burst control packet which can be obtained by sampling the residual offset time.
\end{abstract}

\section{Introduction}

Optical burst switching networks (OBS) is considered as a promising scheme [1] to evolve to all-optical switching networks in future. The burst control packet (BCP) which is sent ahead of burst data's emitting is converted to electronic domain and processed in the control plane. The control plane will incurs queuing delay as well as queuing drop to the BCP due to the congestion and competition from others. So, awareness of the cross traffic intensity of the control plane is significant to the congestion avoidance and performance optimization.

References [2-5] analyzed the impact of the control plane's parameter such as processing rate on the networks performance. Most of these works draw a conclusion that the traffic load on the control plane is a main restriction on the minimum burst length, offset time and the contention probability of the burst. Works in [6-7] gave some burst assembling algorithms that can adjust the threshold of burst length dynamically with the change of the cross traffic on the control plane. Results in above works mainly regard the cross traffic intensity of the control plane as a key parameter to analyze and optimize the network performance. Unfortunately, none of them give an effective way to get it on time. Recently, many end-to-end measurement methods are designed to measure the cross traffic of Internet. They employ some active methods to inject probe traffic into the network which are intrusive and not suitable to the optical links.

In OBS networks, every BCP carries a field named offset time which is used to indicate the upcoming data burst's arrival time. The residual time defined as the field's value detected by egress node can be used to calculate the BCP's end-to-end delay. This delay is equivalent to the sojourn time of the BCP. From [8] we can know that the sojourn time of the BCP is related with the cross traffic in core nodes. Our research presented in this work mainly makes the following two contributions:

- To construct the queuing model of the control plane in core nodes. There are two different models being established depending on whether the wavelength conversion (WC) is present in the core node.

- Develop an estimate method to infer the cross traffic intensity on the control plane of core nodes. The method gets awareness of the cross traffic through the estimator with BCP's sojourn 
time as observing variable.

The rest of the article is organized as follows. Queuing models as well as cross traffic estimators of the control plane are investigated in section II. Section III shows the numerical and simulation results of the estimation. Conclusion and future works are presented in section IV.

\section{Analytical Model}

The queuing behavior of a system is determined mainly by the input traffic character and the service time distribution. The traffic in OBS networks is normally modeled as a Poisson process even though with self-similarity characteristic in packet domain [9]. It is considered that the service time of the core node is just like (1) where $T_{R}$ denotes resource reservation time and $T_{O X C}$ is the configuration time of OXC. The $T_{O X C}$ is determinate for almost all optical switch while the $T_{R}$ is normally stochastic. So the distribution of service time $T_{S}$ is mainly determined by the resource reservation time.

$$
T_{S}=T_{R}+T_{\text {OXC }}
$$

In fact the major work of reservation is to search and fit an optimal void with the arrival burst in order to promote the resource utilization. So the distribution of the number of voids determines that of the reservation time. The reservation time will be determinate if the number of voids to be searched is constant. While reservation time is stochastic if a variable number of voids are searched when carry out the reservation. The following next two subsections discuss the queuing behavior of the control plane with a consideration of WC in core nodes and variant distribution of $T_{R}$. Furthermore two cross traffic estimate methods are proposed based on different queuing model. It is assumed that only one bottleneck core node exists for a flow in OBS networks in the following discussion. It is because that multiple bottleneck nodes can be merged into one queuing system thanks to the independence of the cross traffic in different core nodes. The cross traffic in core nodes is usually mixed with many small flows which are independent each other from many sources. In addition, the flows inject into core nodes along with the switching route of the studied burst is usually different each other. So the independence of the cross traffic with core nodes is reasonable. This assumption is occasionally invalid for example the cross traffic in different core node comes from same flows. The exceptive condition is not considered in our study.

\section{A. Queuing model without WC}

On assumption of no WC device, the resource could only be reserved in the particular wavelength selected by the ingress node. The reservation does not need to search voids because of no other wavelength available. The only work of core nodes is to judge the feasibility of forwarding the burst on the designate wavelength. It requires a simple operation such as only one check or hash operation. Therefore the reservation time is determinate when calculation of the processing unit is based on hardware. The queue then can be modeled as a M/D/1 system.

It is assumed that the traffic intensity on the queue is $\lambda$ bursts/sec in average and the reservation time is $\tau$ (one checking). Then the L-S transform of the PDF of a burst's sojourn time could be presented as (2) [10] where $B(s)$ is the L-S transform of the reservation time and $\rho$ denotes normalized traffic intensity.

$$
W(s)=\frac{s \cdot(1-\rho) \cdot B(s)}{s-\lambda+\lambda \cdot B(s)}
$$

The reservation time's transforming $B(s)$ in (2) is as (3) because of its deterministic distribution.

$$
B(s)=\int_{-\infty}^{\infty} e^{-s t} d B(t)=\int_{-\infty}^{\infty} e^{-s t} \cdot \delta(t-\tau) d t=e^{-s \cdot \tau}
$$

Equation (2) can be rewritten to (4) with the Taylor expansion applied to the $B(s)$ in denominator. 


$$
W(s)=\frac{s(1-\rho) \cdot e^{-s \cdot z}}{s+\lambda \cdot s \cdot \tau \cdot(s \cdot \tau / 2-1)}
$$

The PDF of the sojourn time of bursts in the control plane can be obtained as (5) by applying inverse L-S transform to (4). The coefficient $a(\lambda)$ is shown in (6) where $\mu=1 / \tau$.

$$
\begin{aligned}
& w(t)=a(\lambda) \cdot e^{-a(\lambda) \cdot(t-z)} \\
& a(\lambda)=\frac{2 \cdot \mu \cdot(\mu-\lambda)}{\lambda}
\end{aligned}
$$

An egress node then can make an estimation of the cross traffic in OBS networks given a series end-to-end delay known as $\vec{t}=\left[t_{1} \cdots, t_{n}\right]$. It is easy to get the delay sequence from the residual time carried by the control burst. Egress nodes can construct likelihood function as (7) to estimate the parameter $\lambda$ with $\vec{t}$ as the observing variable. The sampled mean of $\vec{t}$ is denoted by the $\bar{t}$ in the expression.

$$
M(\lambda)=\frac{\sum_{i=1}^{n} \ln w\left(t_{i} \mid \lambda\right)}{n}=\ln a(\lambda)-a(\lambda) \cdot(\bar{t}-1 / \mu)
$$

The estimated parameter $\lambda^{\prime}$ (or the normalized one $\rho^{\prime}$ ) which is the solution of the differential function $M^{\prime}(\lambda)=0$ is drawn in (8).

$$
\lambda^{\prime}=\frac{2 \cdot \mu \cdot(\bar{t} \cdot \mu-1)}{2 \cdot \bar{t} \cdot \mu-1} \text { or } \quad \rho^{\prime}=\frac{2(\bar{t} \cdot \mu-1)}{2 \cdot \bar{t} \cdot \mu-1}
$$

\section{B. Queuing model with WC}

The core node with wavelength conversion device must select an optimal wavelength when scheduling a burst. It requires much more checks and computations rather than the single check in the no-WC situation. The degree of the complexity of reservation algorithm is related to the number of voids encountered when the burst reaches the core nodes. An efficient algorithm in [11] can get $O(\log (N))$ complexity where $N$ is the number of void holding until the data burst's arrival. Investigation from [8] showed that $N$ is usually related with the traffic intensity on the control plane. So, the queuing model of the control plane with WC devices can be constructed as a special $\mathrm{M} / \mathrm{G} / 1$ system. The service time of the queue in this special system stochastically distributed with a PDF function of $\lambda$. Reference [10] gave the PDF of service time as (9) where offset time distributes uniformly in the section $[0, D]$. The symbol $\tau$ in (9) denotes the time of one comparison or hash operation of core nodes. Total reservation time is $N \cdot \tau$ if $N$ voids were seen by the arrival BCP.

$$
P(t=N \cdot \tau)=\frac{(\lambda \cdot D / 2)^{N}}{N !} \cdot e^{-\lambda \cdot D / 2}
$$

It can be seen that the reservation time of core nodes with WC is a discrete stochastic process with Poisson distribution. It is different from a traditional M/G/1 model of which the service time is independent with the input traffic. It is difficult to get the PDF $w(t)$ of the sojourn time because of the intrinsic dependence between the service time and traffic intensity. So, it is difficult to construct a likelihood function analytically like (5). Edge nodes then can only assesses the cross traffic through a method like moment estimator. The mean sojourn time in the M/G/1 queuing model is expressed by (10) from [10].

$$
W=E(t)+\lambda \cdot \frac{\left(1+C_{b}^{2}\right)}{2(1-\rho)}
$$

The $E(t)=\tau \cdot E(N)$ given $N$ voids held in core nodes. Therefore $E(t)=\lambda \cdot D \cdot \tau / 2$ if offset time distributes uniformly in $[0, D]$ from [8]. The square of variance coefficient can be expressed 
as: $C_{b}^{2}=\frac{V^{2}(t)}{[E(t)]^{2}}=\frac{2}{\lambda \cdot D}$ for the Poisson process of the service time. It is worth to emphasize that the normalized traffic load $\rho=\lambda \cdot E(t)=\lambda^{2} \cdot D \cdot \tau / 2$ other than $\rho=\lambda \cdot \tau$ because the service time is not a determinate value but multiple of $\tau$. Finally, the mean sojourn time $W$ in (10) can be rewritten as (11).

$$
, W(\lambda)=\frac{1}{2} \cdot \lambda \cdot D \cdot \tau+\frac{\lambda \cdot D+2}{D \cdot\left(2-\lambda^{2} \cdot D \cdot \tau\right)}
$$

The sojourn time of the burst in the control plane is a discrete value that is multiple of $\tau$ due to the discrete character of the service time. Furthermore the $W(\lambda)$ can be represented as (12) if let $\tau$ be unit time and $D=K \bullet \tau$ where $K$ is a positive integer.

$$
{ } W(\lambda)=\frac{1}{2} \cdot \lambda \cdot K+\frac{\lambda \cdot K+2}{K \cdot\left(2-\lambda^{2} \cdot K\right)}
$$

An egress node will calculates the average end-to-end delay $W^{\prime}$ through the residual offset time value bursts received on time. The cross traffic intensity $\lambda^{\prime}$ then can be estimated by solving the inverse function of (12) assuming $K$ is known.

\section{Numerical and Simulation Result}

We will verify the model's validity through numerical and simulation results in this section. Numerical results are based on the (8) and (12) in section II. Simulation is implemented by C++ tools to emulate a queuing system. The simulation topology is a simple queue model with an ingress node, an egress nodes and a cross traffic generator. The queue is only for the control plane because the data plane has an identical cross traffic as control plane. The main purpose of this work is to obtain the cross traffic from residual offset time which can only be detected from the BCP. Data burst is ignored in the simulation due to its all optical characteristic which can not provide the offset time information. The $\tau$ which is the time of only one check of void table is et to $1 \mu \mathrm{s}$ in the simulation. Furthermore the input traffic from the cross traffic is Poisson. Fig. 1 shows the numerical and simulation results of the estimate model without WC devices. The X-axis of the figure is the sampled mean sojourn time $\bar{t}$ with the multiples of the reservation time $\tau$ as its unit. The simulation result is recorded by the queue in simulation. The model curve is calculated by the egress node in simulation. Fig. 1 reveals that the model is valid because of the no deviation from the simulation result. Furthermore, the extra offset time required to achieve the estimated result is little (about 10 times $\tau$ at most in the figure) because of the determinate service rate.

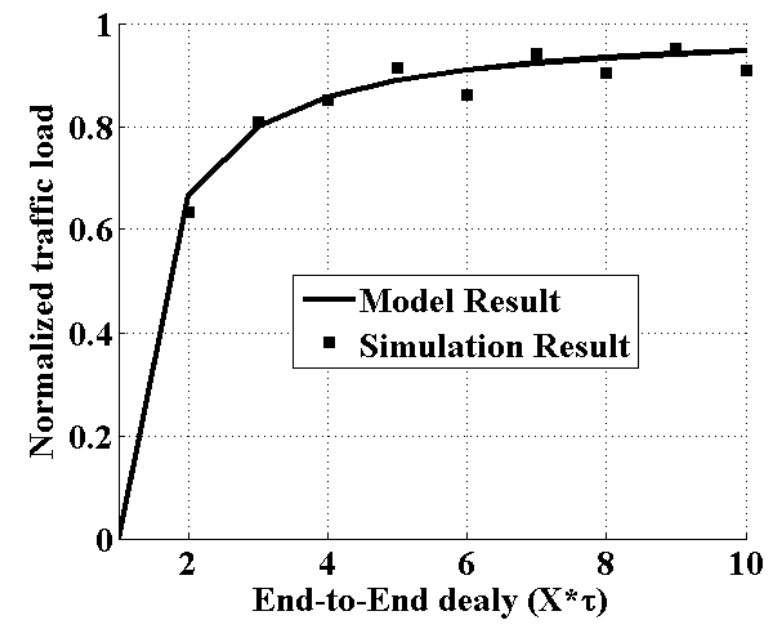

Fig.1 Results without WC

Fig. 2(a) gives a simulation $(K=1)$ and some numerical $(K=[1 . .5])$ results of the core nodes with WC devices. Curves in the figure represent the numerical results. It implies that the upper bound of 
the absolute traffic intensity the control plane can bear is decreasing with the increase of the maximum offset time D. Larger offset time lead to more voids to be searched in reservation. Therefore the service rate of the core plane decreases. While the upper bound of the normalized traffic which is illustrate in Fig. 2(b) is not affected by K. Ellipse in Fig. 2(a) is the simulation result under the condition $K=1$. Simulation result is not consistent with the numerical curve when the traffic load is higher because the buffer in simulation is not infinite. So when the normalized traffic is asymptotical to 1 the queuing time of the queue in simulation is finite. But the results are consistent when the cross traffic is not so intense.

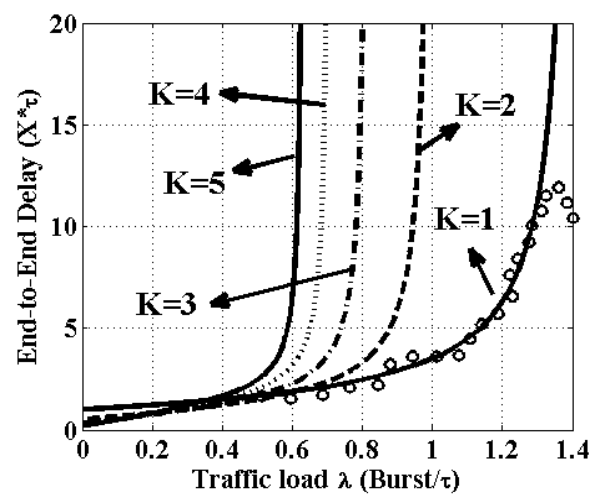

(a) Absolute traffic vs. delay

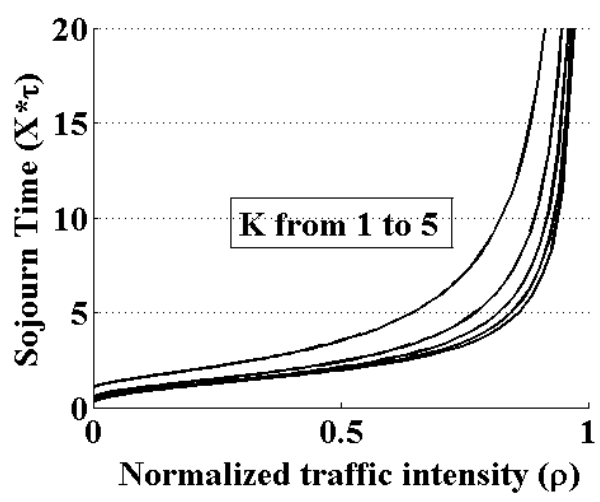

(b) Normalized traffic vs. delay

Fig. 2 Result with WC

\section{Conclusion}

We construct two models to describe the queuing behavior of the control plane of core nodes with WC devices being present or absent. Numerical and simulation results verified the validity of the model. Future works will mainly focus on the dynamic algorithm in edge node to utilize the cross traffic estimated by the model.

\section{References}

[1] Y, Chen, C. Qiao, X. Yu, “Optical burst switching: A new area in optical networking research,” IEEE Netw., vol. 18, pp.16 - 23, mm. 2004.

[2] J. White, M. Zukerman, and H. L. Vu, “A framework fro optical burst switching network design,” IEEE Commun. Lett., vol 6, no. 6, pp. 268 - 270, June 2002.

[3] N. Barakat and T. E. Darcie, "The control-plane stability constraint in optical burst switching networks,” IEEE Commun. Lett., vol. 11, no. 3, pp. 267-269, Mar. 2007.

[4] N. Barakat and T. E. Darcie, "Control plane congestion in optical burst switched networks," IEEE/OSA J. Optical Commun. and Networking, vol.1 no. 3, pp. B98-B110, Aug. 2009.

[5] L. K. Pagaria, T. Venkatesh, C. Siva etc, "Modeling the impact of control plane losses on the performance of optical burst switched core node," IEEE Commun. Lett., vol. 14, no. 11, pp. 1071-1073, November 2010.

[6] M. A, Toksoz, N. Akar, "Dynamic threshold-based assembly algorithms for optical burst switching networks subject to burst rate constraints," Springer Photo Netw Commun. vol. 20, no. 2, pp. 120-130, April 2010.

[7] C. Yuan, Z. Zhang and Z. Li etc, “A unified study of burst assembly in optical burst switching networks,” Springer Photo Netw Commun. vol. 21, no. 5, pp. 228-237, October, 2011. 
[8] L. de Pedro, J. Aracil and J. A. Hernandez etc, "Analysis of the processing and sojourn times of burst control packets in optical burst swithes," in Proc. International Conference on Optical Networks Design and Modeling, pp. 1-3, Mar, 2008.

[9] M. Izal., J. Aracil, “On the influence of self-similarity on optical burst switching traffic,” In Proceeding Globecom. pp. 2308-2312, 2002.

[10]L. Kleinrock, R. Gail, Queuing systems: problems and solutions. L. A.: Wiley-interscience publicatoin, 1996, ch. 5.

[11]J. Xu, C. Qiao and J. Li etc, "Efficient burst scheduling algorithms in optical burst-switched networks using geometric techniques,” IEEE J. Sel on commun, vol. 22, no. 9, pp. 1796-1811, Nov, 2004. 